December 13, 2018

\title{
FIRST PASSAGE PERCOLATION AND ESCAPE STRATEGIES
}

\author{
E. D. ANDJEL AND M.E. VARES
}

\begin{abstract}
Consider first passage percolation on $\mathbb{Z}^{d}$ with passage times given by i.i.d. random variables with common distribution $F$. Let $t_{\pi}(u, v)$ be the time from $u$ to $v$ for a path $\pi$ and $t(u, v)$ the minimal time among all paths from $u$ to $v$. We ask whether or not there exist points $x, y \in \mathbb{Z}^{d}$ and a semi-infinite path $\pi=\left(y_{0}=y, y_{1}, \ldots\right)$ such that $t_{\pi}\left(y, y_{n+1}\right)<t\left(x, y_{n}\right)$ for all $n$. Necessary and sufficient conditions on $F$ are given for this to occur. When the support of $F$ is unbounded, we also obtain results on the number of edges with large passage time used by geodesics.
\end{abstract}

\section{INTRODUCTION}

This work can be motivated by the following game: two individuals, called $\lambda$ and $\sigma$, move on $\mathbb{Z}^{d}$, spending a random time $\tau(e)$ to cross each edge $e=\langle x, y\rangle$ from $x$ to a nearest neighbor site $y$, or vice-versa. These passage times are assumed to be i.i.d. non-negative random variables (further assumptions will be made later). The two individuals start from distinct positions, that we denote by $x_{\lambda}$ and $x_{\sigma}$ respectively; $\lambda$ would like to catch $\sigma$, who in turn wants to escape. A natural and simple question is: knowing the passage times, is $\sigma$ able to devise a strategy that would be successful for his/her goal, independently of what $\lambda$ does? In this case we shall say that $\sigma$ has a perfect strategy. Thus, a perfect strategy is an infinite sequence of moves for $\sigma$ in a way that (s)he will never be caught by $\lambda$ regardless of what (s)he does. We are imagining that the individuals are always sitting on a vertex of $\mathbb{Z}^{d}$ and think of an edge as being a door that remains closed unless someone knocks at it. To open a door corresponding to edge $e$ an individual must be at one of the endpoints of $e$ and knock at it. Once this is done, the door will open after a time interval of length $\tau(e)$, the individual will cross that edge and the door will be closed immediately after. To prevent trivial situations it is natural to assume the common distribution to be useful in the sense of Definition 2.1 below; in particular $\{\tau(e)=0\}$ does not percolate. As we shall see the situation changes significantly depending on the distribution of the passage times being supported on a compact set or not. When $F$ has unbounded support, the probability that a perfect strategy for $\sigma$ exists is zero, independently of the starting positions. On the other hand, in the bounded case and, if a clairvoyant $\sigma$ can chose the initial position depending on $x_{\lambda}$ and the $\tau$ variables, then a perfect strategy can be implemented with probability one. The result is precisely stated in Theorem 2.2 below. The proof in the bounded case is very simple. The unbounded case involves Proposition 3.1, which is the key result of the paper. From it we also derive Theorem 2.3 which states that for $F$ with unbounded support and 
any arbitrary constant $M$, geodesics use asymptotically a positive proportion of edges whose passage times are larger than $M$.

We now outline the paper: in the next section we introduce the basic notation and definitions, and state two theorems. Theorem 2.2 has to do with the above question, and its easy part is proved in the same section. Theorem 2.3 adds information to the comparison of first passage percolation models, treated by van den Berg and Kesten in [1] (in a more general case) in terms of time constants. In section 3 we state and prove Proposition 3.1 which is the main technical result of the paper, from which part (i) of Theorem 2.2 and Corollary 3.2 follow immediately. We then conclude the proofs and discuss a related problem.

\section{Preliminaries AND Results}

\section{Notation and definitions.}

In this paper $\mathbb{E}=\mathbb{E}^{d}$ will denote the set of nearest neighbor (n.n.) edges in the cubic lattice $\mathbb{Z}^{d}$. The origin in $\mathbb{Z}^{d}$ will be denoted by $\mathbf{0}$. For $x, y \in \mathbb{Z}^{d},\|x-y\|$ will denote the $\ell_{1}$-distance, i.e. $\|x\|=\sum_{i=1}^{d}\left|x^{i}\right|$ for $x=\left(x^{1}, \ldots, x^{d}\right) \in \mathbb{Z}^{d}$. A finite path $\pi=\left(e_{1}, \ldots, e_{k}\right)$ is a sequence of adjacent edges (sharing a vertex), i.e. $e_{i}=<x_{i-1}, x_{i}>$ for each $i=1, \ldots, k$. In this case we say that $\pi$ goes from $x_{0}$ to $x_{k}$. For the context of this paper, it suffices to consider self-avoiding paths, i.e. when the $x_{i}, i=0, \ldots, k$ are all distinct, and we always assume this without further comment. Sometimes we identify a path with the sequence of its visited vertices, writing $\pi=\left(x_{0}, \ldots, x_{k}\right)$.

The basic random object consists of a family $\{\tau(e): e \in \mathbb{E}\}$ of i.i.d. non-negative random variables defined on a probability space $(\Omega, \mathcal{F}, P)$, where $\tau(e)$ represents the passage time of the edge $e$, interpreted as the time to traverse $e$. Their common distribution will be denoted by $F$. The passage time $t(\pi)$ of a given path $\pi=\left(e_{1}, \ldots, e_{k}\right)$ is simply given by the sum of the variables $\tau\left(e_{i}\right)$ for $i=1, \ldots, k$. We say that a given path $\tilde{\pi}$ from $x$ to $y$ is optimal (from $x$ to $y$ ) if its travel time is the shortest among all paths from $x$ to $y$ :

$$
t(\tilde{\pi})=\inf \{t(\pi): \pi \text { is a path from } x \text { to } y\}=: t(x, y) .
$$

Any such optimal path is also called a geodesic (from $x$ to $y$ ). An infinite path $\tilde{\pi}=\left(e_{1}, e_{2}, \ldots\right.$ ) starting at $x$ is said to be a semi-infinite geodesic if for any $n$ the finite path $\left(e_{1}, \ldots, e_{n}\right)$ is a geodesic from $x$ to its endpoint. It is easy to see that semi-infinite geodesics starting from any given point always exist. We also see easily that when $F$ is continuous, there is a.s. a unique optimal path from $x$ to $y$ for any two distinct vertices $x$ and $y$. Here, some assumptions on $F$ will be needed, and as in [1] we set the following:

Definition 2.1. A distribution $F$ with support in $[0,+\infty)$ is called useful if the following holds:

$$
\begin{aligned}
& F(r)<p_{c} \text { when } r=0, \\
& F(r)<\overrightarrow{p_{c}} \text { when } r>0,
\end{aligned}
$$


where $p_{c}\left(\overrightarrow{p_{c}}\right)$ denotes the critical probability for the Bernoulli (oriented, resp.) bond percolation model on $\mathbb{Z}^{d}$, and $r$ stands for the minimum of the support of $F$, hereby denoted by $\operatorname{supp}(F)$.

Theorem 2.2. Let $F$ be useful in the sense of Definition 2.1.

(i) If $F$ has unbounded support, then for any $x_{\lambda}, x_{\sigma}$

$$
P(\sigma \text { has perfect strategy })=0 \text {. }
$$

(ii) Assume $F$ to be supported in $[0, M]$ for some finite $M$. Let $\tilde{\pi}$ be a semi-infinite geodesic from $x_{\lambda}$. If the event

$$
\left[M+t\left(x_{\sigma}, x\right)<t\left(x_{\lambda}, x\right) \text { for some } x \in \tilde{\pi}\right]
$$

occurs, then $\sigma$ has a perfect strategy. In particular, given $x_{\lambda}$, with probability one there exist (infinitely many) random initial positions $x_{\sigma}$ from where $\sigma$ has a perfect strategy.

Proof. Part (i) will be proven in Section 3 as a corollary of our Proposition 3.1. We now prove only the easy part (ii). Indeed, under the situation described in (2.4), it follows at once that a perfect strategy for $\sigma$ consists in taking any $x \in \tilde{\pi}$ for which $M+t\left(x_{\sigma}, x\right)<t\left(x_{\lambda}, x\right)$, moving to $x$ by the geodesics from $x_{\sigma}$ to $x$ and then following the infinite branch of $\tilde{\pi}$ that starts in $x$. On the other hand, if $F$ is useful it follows at once from the definitions that there exists $\delta>0$ so that $F(\delta)<p_{c}$, which implies $t\left(x_{\lambda}, x\right) \rightarrow \infty$ as $x \rightarrow \infty$ along $\tilde{\pi}$, and the inequality in (2.4) becomes trivial for $x_{\sigma} \in \tilde{\pi}$ with $t\left(x_{\lambda}, x_{\sigma}\right)>M$.

We now state our second theorem.

Theorem 2.3. Let $F$ be a useful distribution on $[0, \infty)$ with unbounded support. Then, for each $M$ positive there exists $\epsilon=\epsilon(M)>0$ and $\alpha=\alpha(M)>0$ so that for all $n \geq 1$ and all $x$ with $\|x\|=n$, we have

$$
P\left(\exists \text { geodesic } \pi \text { from } 0 \text { to } x \text { such that } \sum_{e \in \pi} \mathbf{1}_{\{\tau(e)>M\}} \leq \alpha n\right) \leq e^{-\epsilon n} .
$$

\section{BAsic proposition. Proofs.}

Proposition 3.1. Let $F$ be a useful distribution on $[0, \infty)$ with unbounded support. For each $M>0$ let

$$
\bar{t}_{(M)}(\mathbf{0}, x)=\inf \{t(\pi): \pi \text { is a path from } 0 \text { to } x \text { with } \tau(e) \leq M \text { for all } e \text { in } \pi\},
$$

with the understanding that inf $\emptyset=+\infty$. Then, for each $M$ positive there exists $\epsilon=\epsilon(M)>0$ and $n_{0}=n_{0}(M)$ so that for all $n \geq n_{0}$ and all $x$ such that $\|x\|=n$, we have

$$
P\left(M+t(\mathbf{0}, x)<\bar{t}_{(M)}(\mathbf{0}, x)\right) \geq 1-e^{-\epsilon n} .
$$

The proof of the above proposition uses arguments from [1] that are recalled below. Before moving to it, we state the following immediate consequence: 
Corollary 3.2. Let $F$ be a useful distribution on $[0, \infty)$ with unbounded support. Then, for each $M$ positive there exists $\epsilon=\epsilon(M)>0$ so that for all $n \geq 1$ and all $x$ with $\|x\|=n$, we have

$$
P(\exists \text { geodesic } \pi \text { from } \mathbf{0} \text { to } x \text { such that } \tau(e) \leq M \text { for all } e \in \pi) \leq e^{-\epsilon n} \text {. }
$$

Notation. For $N \in \mathbb{N}$ and $l=\left(l^{1}, \ldots, l^{d}\right) \in \mathbb{Z}^{d}$ consider the following partition of $\mathbb{Z}^{d}$ by hypercubes, as in [1] called $N$-cubes.

$$
S_{l}(N)=\left\{x \in \mathbb{Z}^{d}: N l^{i} \leq x^{i}<N l^{i}+N, \forall i\right\} .
$$

The cubes are naturally indexed by $l$, and this indexing is also used to define the distance between two $N$-cubes. If $C \subset \mathbb{Z}^{d}$, we use $\mathcal{F}(C)$ to denote the $\sigma$-field generated by the variables $\tau(e)$ corresponding to edges $e$ that have both endpoints in the set $C$.

The following collections of boxes $T_{l}(N), B_{l}^{+j}(N)$ and $B_{l}^{-j}(N)$ will also be useful in the proofs: for $N \in \mathbb{N}, l \in \mathbb{Z}^{d}$,

$$
\begin{array}{r}
T_{l}(N)=\left\{x \in \mathbb{Z}^{d}: N l^{i}-N \leq x^{i} \leq N l^{i}+2 N, \forall i\right\} . \\
B_{l}^{ \pm j}(N)=T_{l}(N) \cap T_{l \pm 2 \mathbf{e}_{j}}(N), \quad j=1, \ldots, d,
\end{array}
$$

where $\mathbf{e}_{j}, j=1, \ldots, d$ denote the canonical unitary vectors.

We first recall Lemma (5.2) from [1] (see also [3]) which follows from a Peierls argument:

Lemma 3.3. If the cubes $S_{l}(N)$ are colored black or white in a random fashion which is (i) translation invariant; (ii) finite range (i.e. the color of $S_{l}(N)$ is $\mathcal{F}\left(\cup_{l^{\prime}}\left(S\left(l^{\prime}, N\right):\left\|l^{\prime}-l\right\| \leq c_{0}\right)\right.$ measurable for a suitable constant $\left.c_{0}\right)$ and moreover, $P\left(S_{0}(N)\right.$ is black $) \rightarrow 1$ as $N \rightarrow \infty$, then for all $N$ sufficiently large we can find positive numbers $\epsilon=\epsilon(N)$ and $D=D(N)$ so that for each $u, v \in \mathbb{Z}^{d}$ the probability that each path from $u$ to $v$ visits at least $\epsilon\|u-v\|$ distinct black $N$-cubes is not smaller than $1-e^{-D\|u-v\|}$.

We must also recall Lemma (5.5) from [1], which says that if the distribution $F$ of the time variables is useful, then there exist positive numbers $\delta=\delta(F)$ and $D_{0}=D_{0}(F)$ such that

$$
P(t(u, v) \leq(r+\delta)\|u-v\|) \leq e^{-D_{0}\|u-v\|},
$$

for all $u, v \in \mathbb{Z}^{d}$, where $r$ is as in Definition 2.1 .

Although it is assumed troughout [1] that $F$ has finite first moment, this requirement is not used in the proof of (3.6).

Of course, for the proof of the proposition it suffices to consider $M>0$ large and such that $P(\tau(e) \in(M, M+1])>0$. In the proof we shall also consider optimal paths for the passage times

$$
\bar{\tau}(e)= \begin{cases}\tau(e) & \text { if } \tau(e) \leq M \\ +\infty & \text { otherwise }\end{cases}
$$


Black cubes. We take $\delta=\delta(F)$ and $D_{0}=D_{0}(F)$ so that (3.6) holds. Let $M \in(0,+\infty)$. We now say that the $N$-cube $S_{l}(N)$ is black if for any path $\pi$ lying entirely in $T_{l}(N)$ with endpoints $u, v$ such that $\|u-v\| \geq N / 4$ and using only edges with passage times less than or equal to $M$, we do have $t(\pi) \geq(r+\delta)\|u-v\|$. The $N$-cubes $S_{l}(N), S_{l^{\prime}}(N)$ are said to be separated if $T_{l}(N) \cap T_{l^{\prime}}(N)=\emptyset$.

From (3.6) we see that Lemma 3.3 applies. In particular, having fixed $\delta$ as above, for any $N$ sufficiently large we can take $D=D(N, F)>0$ and $\epsilon=\epsilon(N, F)>0$ in a way that for all $n$ large enough:

$P\left(\exists\right.$ path from 0 to $\Gamma_{n}$ that visits at most $[\epsilon n]$ separated black $N-$ cubes $\} \leq e^{-D n}$,

where $\Gamma_{n}=\left\{x \in \mathbb{Z}^{d}:\|x\|=n\right\}$ and [.] denotes the integer part. (Of course, changing $D$ we may assume (3.7) holds for all $n$.)

We shall now work on the complement of the event on the 1.h.s. of (3.7). For the proof of Proposition 3.1 we will try to improve over the optimal paths for $\bar{\tau}$ from 0 to some $x$ in $\Gamma_{n}$ by examining the probability of successful shortcuts in disjoint boxes $B_{l}^{ \pm j}(N)$. The main point is the control of the conditional probability of a successful shortcut.

Definition 3.4. We say that a path $\pi$ crosses the box $B_{l}^{ \pm j}(N)$ if it crosses the box in the shortest direction and, except for its endpoints, is entirely contained in the interior of $B_{l}^{ \pm j}(N)$.

In the sequel we write $\pi_{[u, v]}$ to denote the stretch of $\pi$ starting at $u$ and ending at $v$.

Definition 3.5. We say that a stretch $\pi_{[u, v]}$ of $\pi$ is shortcutable if it crosses one of the boxes $B_{l}^{ \pm j}(N)$ corresponding to a black cube $S_{l}(N)$.

Definition 3.6. For $\rho>0$ and small, we say that a path from the origin to $\Gamma_{n}$ satisfies property $\mathcal{P}_{n}(\rho)$ if it contains at least $[\rho n]$ (integer part of $\rho n$ ) shortcutable stretches which lie at distance at least $14 N$ of each other.

Lemma 3.7. Let $N$ be large enough for (3.7) to hold. There exist constants $\rho=\rho(N, F)>0$ and $D=D(N, F)>0$ such that for all $n$ the probability that all paths from the origin to $\Gamma_{n}$ satisfy condition $\mathcal{P}_{n}(\rho)$ is at least $1-\exp (-D n)$.

Proof. It is clear that if $\pi$ is a path connecting a vertex $x$ in $S_{l}(N)$ to $y \notin T_{l}(N)$, it must contain a path that crosses one of the $2 d N$-boxes $B_{l}^{ \pm j}(N)$ in the sense just defined. Hence the lemma follows at once from (3.7). 


\section{Shortcuts}

Let $\pi$ be a path from 0 to a point in $\Gamma_{n}$ whose edges have passage times less than or equal to $M$. Let $\pi^{\prime}$ be a shortcutable stretch of $\pi$ and call $B$ the $N$-box (corresponding to a black cube) it crosses. Assuming $\pi$ to be optimal for the $\bar{\tau}$ variables, we shall examine the possibility of a successful shortcut $\tilde{\pi}$ for $\pi$ that uses an edge with passage time larger than $M$. This would be a path verifying the following conditions:

- $\tilde{\pi}$ and $\pi$ are edge disjoint;

- the endpoints of $\tilde{\pi}$ coincide with those of a segment $\pi^{\prime \prime}$ of $\pi$;

- $|\tilde{\pi}| \leq c_{d} N$ where the positive constant $c_{d}$ depends only on the dimension;

- $\tilde{\pi}$ is contained in the same $N$-box $B$ as $\pi^{\prime}$.

We shall then say that a shortcut as above is successful if $M+t(\tilde{\pi})<t\left(\pi^{\prime \prime}\right)$.

Let us first assume for notational simplicity that $d=2, B=B_{l}^{1}(N)$, which we write as $B=[a, a+N] \times[b, b+3 N]$, and that $\pi^{\prime}$ crosses $B$ from left to right. Writing $\pi=\left(x_{0}, \ldots, x_{s}\right)$, let $v=x_{j}$ be the position in $\{a+N\} \times[b+1, b+3 N-1]$ where $\pi$ first reaches the rightmost face of $B$ after entering $B$ and $u=x_{i}$ the position in $\{a\} \times[b+1, b+3 N-1]$ of the leftmost face of $B$ last visited before getting to $v$, so that $i<j$ and $\pi^{\prime}$ is the segment of $\pi$ that goes from $u$ to $v$, which we denote as $\pi_{[u, v]}$. We choose $N=4 K$ for some $K \in \mathbb{N}$. We may define as well the vertex with lowest second coordinate and first coordinate in $[a+K, a+3 K]$ along $\pi_{[u, v]}$. If there are several such points, let us take e.g. the leftmost one, call it $z=\left(z^{1}, z^{2}\right)$. We assume that $z$ is on the leftmost half of $B$, i.e. $z^{1} \leq a+N / 2$ (the argument being analogous when $z$ is on the rightmost half of $B$ ); we now define $\tilde{\pi}$ by starting from $z$ moving downwards one step to $z^{\prime}=z-\mathbf{e}_{2}$ and then moving horizontally to the right for at most $K$ steps or until we reach any point $w$ in $\pi$, whatever comes earlier (note that we can have $w=z^{\prime}$ ). In the first case, we then move vertically upwards until reaching a vertex $w$ visited by $\pi$; this just defined path from $z$ to $w$ is what we call $\tilde{\pi}$. Three cases have to be analyzed:

(a) $w \in \pi_{[u, v]}$,

(b) $w$ is visited by $\pi$ before $u$,

(c) $w$ is visited by $\pi$ after $v$.

In all of these three cases we define a new path substituting the stretch of $\pi$ between $w$ and $z$ by $\tilde{\pi}$

In case (a) the substituted part is the stretch $\pi_{[z, w]}$ contained in $\pi_{[u, v]}$. In this case $\|z-w\| \geq$ $K$.

In case (b) the substituted part is the portion of $\pi$ going from $w$ to $z$.

In case (c) the substituted part is the portion of $\pi$ going from $z$ to $w$.

It is easy to check that in all three cases the substituted part of $\pi$ contains a stretch of $\pi_{[u, v]}$ connecting two points at distance at least $K=N / 4$. If $\pi_{[u, v]}$ is shortcutable, then the time of the substituted part is at least $\|z-w\| r+K \delta$.

The extension to higher dimension is simple and we always have $\|z-w\| \leq 3 N d=12 K d$. Assuming that the stretch $\pi_{[u, v]}$ is shortcutable, a condition which guarantees a successful shortcut is $M+\sum_{e_{i} \in \tilde{\pi}} \tau\left(e_{i}\right)<\|z-w\| r+K \delta$. Note that the number of edges in $\tilde{\pi}$ is at most $\|z-w\|+2$. For our application below (proof of Proposition 3.1), we shall impose for one 
of the edges, call it $e_{1}$, that $\tau\left(e_{1}\right) \in(M, M+1)$ and for the other edges we impose passage times in the interval $\left[r, r+\delta^{\prime}\right)$ with $\delta^{\prime}=\delta /(24 d)$. Hence, the shortcut is successful if

$$
2 M+1+(\|z-w\|+1)\left(r+\delta^{\prime}\right)<\|z-w\| r+K \delta
$$

which is implied by

$$
2 M+1+r+\delta /(24 d)+K \delta / 2<K \delta
$$

and this, in its turn, is satisfied for $K$ large enough (depending on $M, \delta$ and $r$ ).

\section{Proof of Proposition 3.1.}

Let $\delta$ be as in the definition of black cubes and take $M>0$ so that $P(\tau \in(M, M+1])>0$. We fix $N=4 K$ large so that the conclusion of Lemma 3.7 holds and moreover $2 M+1+$ $r+\delta /(24 d)<K \delta / 2$ as in the above construction. We may as well assume that the set on the right side of (3.1) is not empty, and let $\Pi$ be a path where the minimum is attained. (In case of non-uniqueness, the argument will apply to any of the finitely many optimal paths, and any deterministic way to list them will do the job.)

We now define random variables $U_{1}, V_{1}, U_{2}, V_{2}, \ldots$ taking values in $\mathbb{Z}^{d} \cup\{\infty\}$. On the event $\{\Pi=\pi\}, U_{1}, V_{1}$ are such that $\pi_{\left[U_{1}, V_{1}\right]}$ is the first shortcutable stretch of $\pi$. If no such stretch exists then $U_{1}=V_{1}=\infty ; U_{2}, V_{2}$ are such that $\pi_{\left[U_{2}, V_{2}\right]}$ is the first shortcutable stretch of $\pi$ after $V_{1}$ whose distance to $\pi_{\left[U_{1}, V_{1}\right]}$ is at least $7 N$. In general, $U_{i+1}, V_{i+1}$ is such that $\pi_{\left[U_{i+1}, V_{i+1}\right]}$ is the first shortcutable stretch of $\Pi$ after $V_{i}$ whose distance to $\cup_{j=1}^{i} \Pi_{\left[U_{j}, V_{j}\right]}$ is at least $7 N$, or $U_{i+1}=V_{i+1}=\infty$ if no such stretch exists.

For a given $n$ let $q=q(n)=[\rho n]$. Then, partition the probability space in events as $A\left(\pi, x_{1}, y_{1}, \ldots, x_{q}, y_{q}\right)=\left\{\Pi=\pi, U_{i}=x_{i}, V_{i}=y_{i}: 1 \leq i \leq q\right\}$ and the event $G=\left\{U_{q}=\right.$ $+\infty\}$. For each of the shortcutable stretches of $\pi$ there is a path $\tilde{\pi}_{i}$ as defined above, with $z_{i}, w_{i}$ the corresponding vertices in that construction.

Call $e_{i, 1}, \ldots, e_{i, k_{i}}$ the edges of $\tilde{\pi}_{i}$ and call $e_{i, 1}^{\prime}, \ldots, e_{i, \ell_{i}}^{\prime}$ the edges which have one endpoint in $\tilde{\pi}_{i} \backslash\left\{w_{i}, z_{i}\right\}$ and whose other endpoint is not in $\tilde{\pi}_{i}$. We now define the event

$$
\begin{gathered}
F_{i}\left(\pi, x_{1}, y_{1}, \ldots, x_{q}, y_{q}\right)=A\left(\pi, x_{1}, y_{1}, \ldots, x_{q}, y_{q}\right) \cap \\
\left\{\tau\left(e_{i, 1}\right) \in(M, M+1], \tau\left(e_{i, 2}\right)<r+\delta^{\prime}, \ldots, \tau\left(e_{i, k_{i}}\right)<r+\delta^{\prime}, \tau\left(e_{i, 1}^{\prime}\right)>M, \ldots, \tau\left(e_{i, \ell_{i}}^{\prime}\right)>M\right\},
\end{gathered}
$$

with $\delta^{\prime}=\delta /(24 d)$ as before. Notice that $k_{i}, \ell_{i}$ are uniformly (in $i$ ) bounded by a constant that depends only on $K$ and $d$.

If the event $F_{i}\left(\pi, x_{1}, y_{1}, \ldots, x_{n}, y_{n}\right)$ occurs then substituting a part of $\pi$ as explained before we get a new path $\pi_{i}^{\prime}$, and $M+t\left(\pi_{i}^{\prime}\right)<t(\pi)$.

Since by Lemma $3.7 P(G) \leq e^{-D n}$, to conclude the proof it suffices to show that

$$
P\left(\cap_{i=1}^{q} F_{i}^{c}\left(\pi, x_{1}, y_{1}, \ldots, x_{q}, y_{q}\right) \mid A\left(\pi, x_{1}, y_{1}, \ldots, x_{q}, y_{q}\right)\right) \leq(1-\varepsilon)^{q}
$$

for some $\varepsilon>0$ (independent of $q$ ).

The proof of (3.10) will follow by a suitable application of the following simple lemma.

Lemma 3.8. Let $\Omega=\mathbb{R}^{\Lambda}$, where $\Lambda$ is a finite or countable set, endowed with the usual product Borel sigma-field $\sigma(\Lambda)$. Let $\Lambda_{1}$ be a (non-empty) finite proper subset of $\Lambda$ and $\Lambda_{2}=\Lambda \backslash \Lambda_{1}$. 
For $i=1,2$ let $\Omega_{i}=\mathbb{R}^{\Lambda_{i}}$, so that $\Omega=\Omega_{1} \times \Omega_{2}$ and $\sigma(\Lambda)=\sigma\left(\Lambda_{1}\right) \times \sigma\left(\Lambda_{2}\right)$. Let $\mu_{i}$ be $a$ Borel probability measure on $\left(\Omega_{i}, \sigma\left(\Lambda_{i}\right)\right), i=1,2$ and $\mu=\mu_{1} \times \mu_{2}$ the product measure on $(\Omega, \sigma(\Lambda))$. If $A \in \sigma(\Lambda)$ and $\hat{B} \in \sigma\left(\Lambda_{1}\right)$ have the property that $x=\left(x_{1}, x_{2}\right) \in A$ and $y_{1} \in \hat{B}$ imply $\left(y_{1}, x_{2}\right) \in A$, then

$$
\mu(B \cap A) \geq \mu(B) \mu(A)
$$

where $B=\hat{B} \times \Omega_{2}$.

Proof. The hypothesis on $A$ and $\hat{B}$ can be written as

$$
\mathbf{1}_{\hat{B}}\left(y_{1}\right) \mathbf{1}_{A}\left(x_{1}, x_{2}\right) \leq \mathbf{1}_{\hat{B}}\left(y_{1}\right) \mathbf{1}_{A}\left(y_{1}, x_{2}\right)
$$

for all $x_{1}, y_{1} \in \Omega_{1}$ and all $x_{2} \in \Omega_{2}$. We compute the iterated integral $\mu_{1}\left(d y_{1}\right) \mu_{1}\left(d x_{1}\right) \mu_{2}\left(d x_{2}\right)$ on both sides. The left hand side yields, by Tonelli's theorem, $\mu(B) \mu(A)$. On the right hand side we have

$$
\int_{\Omega_{1}} \mu_{1}\left(d y_{1}\right) \int_{\Omega_{1}} \mu_{1}\left(d x_{1}\right) \int_{\Omega_{2}} \mu_{2}\left(d x_{2}\right) \mathbf{1}_{\hat{B}}\left(y_{1}\right) \mathbf{1}_{A}\left(y_{1}, x_{2}\right)
$$

which again by Tonelli's theorem can be rewritten as

$$
\int_{\Omega} \mu\left(d y_{1}, d x_{2}\right) \mathbf{1}_{B \cap A}\left(y_{1}, x_{2}\right) \int_{\Omega_{1}} \mu_{1}\left(d x_{1}\right)=\mu(B \cap A)
$$

proving the lemma.

To conclude the proof of Proposition 3.1, let

$$
A=A\left(\pi, x_{1}, y_{1}, \ldots, x_{q}, y_{q}\right)=\left\{\Pi=\pi, U_{i}=x_{i}, V_{i}=y_{i}: 1 \leq i \leq q\right\} \text { and }
$$

$\hat{B}_{i}=\left\{\tau\left(e_{i, 1}\right) \in(M, M+1], \tau\left(e_{i, 2}\right)<r+\delta^{\prime}, \ldots, \tau\left(e_{i, k_{i}}\right)<r+\delta^{\prime}, \tau\left(e_{i, 1}^{\prime}\right)>M, \ldots, \tau\left(e_{i, \ell_{i}}^{\prime}\right)>M\right\}$,

for $i=1, \ldots, q$, so that $P\left(\hat{B}_{i}\right) \geq \eta>0$ for all $i$. A few instants of reflection show that the condition in the lemma is verified for the pair $A$ and $\hat{B}_{1}$ : since $\Pi$ is optimal for the $\bar{\tau}$ variables and has a finite time, it cannot cross any of the edges $e_{1,1}^{\prime}, \ldots, e_{1, \ell_{1}}^{\prime}$; this prevents it from using the advantageous edges $e_{1,2}, \ldots, e_{1, k_{1}}$, and therefore the modified configuration remains in $A$. Call $F_{i}$ the event defined in (3.9). Since $F_{1}=A \cap \hat{B}_{1}$, the lemma implies that $P\left(F_{1}^{c} \mid A\right) \leq 1-P\left(\hat{B}_{1}\right)$. Analogously, we can again apply the lemma with $A$ replaced by $A \cap \cap_{j=1}^{i-1} F_{j}^{c}$ for $i=2, \ldots, q$ and $\hat{B}_{i}$ to conclude that conditional probability on the l.h.s. of $(3.10)$ is bounded from above by $(1-\eta)^{[q n]}$.

Remark 3.9. The argument used to prove Proposition 3.1 also shows that under the same conditions, for each $M$ positive there exist $\alpha>0$ and $\epsilon>0$ (both depending on $M$ ) so that for all $n \geq 1$ and all $x \in \Gamma_{n}$, we have

$$
P\left(t(\mathbf{0}, x)+\alpha n<\bar{t}_{(M)}(\mathbf{0}, x)\right) \geq 1-e^{-\epsilon n} .
$$


Recall now the time constant associated to the passage time distribution $F$, given by the deterministic limit (in probability):

$$
\mu_{F}=\lim _{n \rightarrow \infty} \frac{1}{n} t\left(\mathbf{0}, n \mathbf{e}_{1}\right) .
$$

The limit is also a.s. and in $L_{1}$ under conditions on the tail of $F$, e.g. if $F$ has finite mean, and also holds along any fixed direction, not only the coordinate axis. (See $[6,2,4,5]$.) When $F$ has exponentially decaying tail, the result in Remark 3.9 , taking e.g. $x=n \mathbf{e}_{1}$, can be seen as consequence of large deviation estimates on the variables $t(0, x) / n$. (See e.g. [4].)

Proof of part (i) of Theorem 2.2.

It is clear that with probability one, no perfect strategy for $\sigma$ can consist in remaining in a finite set for all times and so it must reach the set $\left\{x:\left\|x-x_{\sigma}\right\|=n\right\}$ for any $n$. It is obvious that on the event $\left\{t\left(x_{\lambda}, x_{\sigma}\right)<M\right\}$ any perfect strategy can only use edges with passage time smaller than $M$ and cannot include finite paths between two points whose passage time exceeds the minimal passage time between these points by more than $M$. Thus

$P\left(\sigma\right.$ has perfect strategy, $\left.t\left(x_{\lambda}, x_{\sigma}\right)<M\right) \leq P\left(\exists x:\left\|x-x_{\sigma}\right\|=n, \bar{t}_{(M)}\left(x_{\sigma}, x\right) \leq M+t\left(x_{\sigma}, x\right)\right)$

Given $\eta>0$, let $M$ be such that $P\left(t\left(x_{\lambda}, x_{\sigma}\right) \geq M\right) \leq \eta$. Given such $M$ we take $n$ and $\epsilon$ so that (3.2) holds and $c_{d} n^{d-1} e^{-\epsilon n} \leq \eta$, where $c_{d} n^{d-1}$ is an upper bound for the cardinality of $\{x:\|x\|=n\}$. We then have

$$
P(\sigma \text { has perfect strategy }) \leq 2 \eta \text {. }
$$

Proof of Theorem 2.3. An $N$-cube $S_{l}(N)$ is now colored black if every geodesic from a point on its boundary to a point on the boundary of $T_{l}(N)$ uses at least one edge whose passage time is larger than $M$. From Corollary 3.2 we see that Lemma 3.3 applies, and the result follows.

Remark 3.10. The same argument used for Theorem 2.3 yields the following result: Let A be a Borel set to which $F$ attributes positive measure. Then,

$$
P\left(\exists \text { geodesic } \pi \text { from } \mathbf{0} \text { to } x \text { such that } \sum_{e \in \pi} \mathbf{1}_{\{\tau(e) \in A\}} \leq \alpha n\right) \leq e^{-\epsilon n} .
$$

This last result improves inequality (2.16) in [1].

An open problem. Suppose that we have a family of independent Poisson processes $\left\{\mathcal{P}_{e}: e \in \mathbb{E}^{d}\right\}$ of parameter $c>0$. Assume that the individuals $\lambda$ and $\sigma$ may now move from a vertex $x$ to a n.n. vertex $y$ at the jump times of $\mathcal{P}_{e}$ where $e=\langle x, y\rangle$. In dimension one, as easily seen, the probability that $\sigma$ has a perfect strategy is zero. What happens in higher dimensions?

Acknowledgement. We warmly thank a referee who showed us that Theorem 2.3 could be derived from Corollary 3.2. E.D.A. thanks the kind hospitality of IMPA, Rio de Janeiro 
during the preparation of this paper. The work of E.D.A. was partially supported by CNRS and by CAPES. M.E.V is partially supported by CNPq grant 304217/2011-5 and project 474233/2012-0. Most of this work was done while M.E.V. was researcher of CBPF.

\section{REFERENCES}

[1] J. van den Berg and H. Kesten (1993) Inequalities for the time constant in first-passage percolation. Ann. Applied Probab. 3, 56-80.

[2] J. T. Cox and H. Kesten (1981) On the continuity of the time constant of first passage percolation. $J$. Appl. Probab. 18, 809-819.

[3] G. Grimmett and H. Kesten (1984) First passage percolation, network flows and electrical resistances. Z. Wahrsch. Verw. Gebiete 66 335-366.

[4] H. Kesten (2003) First passage percolation. In: From classical to modern probability: CIMPA Summer School 2001. Eds. Pierre Picco, Jaime San Martin. Progress in Probability 54, 93-143.

[5] R. Marchand (2002) Strict inequalities for the time constant in first passage percolation. Ann. Appl. Probab. 12, 1001-1038.

[6] D. Richardson (1973) Random growth in a tessellation. Proc. Cambridge Philos. Soc. 74, 515-528.

Enrique D. AndJel, LATP URA 225/CNRS,

UniVERsité D'AiX-MARSEILle,

39, rue Joliot Curie. 13453 Marseille cedex 13, France

E-mail address: Enrique.Andjel@cmi.univ-mrs.fr

Maria Eulalia Vares, DMe, Instituto de Matemática, Universidade Federal do Rio de Janeiro, Av. Athos da Silveira Ramos 149, CEP 21941-909 - Rio de Janeiro, RJ, Brasil E-mail address: eulalia@im.ufrj.br 